\title{
Wetting Transition Energy Curves for a Droplet on a Square-Post Patterned Surface
}

4
5

2

Abstract: Due to the property of water repellence, biomimetic superhydrophobic surfaces have been widely applied to green technologies, in turn inducing wider and deeper investigations on superhydrophobic surfaces. Theoretical, experimental and numerical studies on wetting transitions have been carried out by researchers, but the mechanism of wetting transitions between Cassie-Baxter state and Wenzel state, which is crucial to develop a stable superhydrophobic surface, is still not fully understood. In this paper, the free energy curves based on the transition processes are presented and discussed in detail. The existence of energy barriers with or without consideration of the gravity effect, and the irreversibility of wetting transition are discussed based on the presented energy curves. The energy curves show that different routes of the Cassie-to-Wenzel transition and the reverse transition are the main reason for the irreversibility. Numerical simulations are implemented via a phase field lattice Boltzmann method of large density ratio, and the simulation results show good consistency with the theoretical analysis.

\footnotetext{
Keywords: wetting transition, energy curves, lattice Boltzmann method
} 
$55=r \cos \theta_{Y}$ [7].

\section{Introduction}

Surface roughness, which can be found in the form of micro or hierarchical structures in nature, has been widely investigated for its enhancement to hydrophobicity [1-4]. Through mimicking natural superhydrophobic surfaces including plant leaves and animals such as lotus leaves, rice leaves and water strider legs, manmade superhydrophobic surfaces via various of methodologies have been presented and applied in industrial applications, for instance, coating, self-cleaning surfaces, microfluidic devices with surface-tension-induced drop motion and so forth $[5,6]$. Among all the natural water-repellence examples, lotus leaves are the most impressive for their superhydrophobic characteristic which is also known as "lotus effect". Due to the micrometre order length scales of the micro posts on the surfaces, the apparent contact angle (APCA) of lotus leaves is approximately $160^{\circ}$ while the hysteresis angle is just about $4^{\circ}$

As the wetting phenomena have been investigated over the past decades, significant progress on theoretical models has also been achieved with considerable attention. The starting point of wetting on an ideal rigid, flat and homogeneous surface is characterized by the well-known Young's Equation [8]:

$$
\cos \theta_{Y}=\frac{\sigma_{S G}-\sigma_{S L}}{\sigma_{L G}}
$$

where $\sigma$ is the surface tension which represents the energy per unit area of the interface between solid/gas, solid/liquid or liquid/gas, and $\theta_{Y}$ is the Young's contact angle. Young's Equation reveals the relationship between surface tensions and contact angle in the ideal situation, however, it cannot be applied to most real surface conditions due to the existence of surface roughness. For the surface roughness, a new correlation where the apparent contact angle is related to surface roughness was presented by Wenzel [9]:

$$
\cos \theta_{w}=r \frac{\sigma_{S G}-\sigma_{S L}}{\sigma_{L G}}
$$

which is also normally written as the following reformed equation: 
where $r$, the roughness parameter corresponding to the "roughness factor", which is also referred to as roughness area ratio, denotes as the ratio of the actual surface area with respect to the projected structure surface, and $\theta_{w}$ is the Wenzel's angle. The Wenzel equation is associated with the homogeneous wetting states, where the grooves caused by the surface roughness are penetrated with water. Apart from the homogeneous wetting state, there is another stable state, the heterogeneous wetting state, and the corresponding equation to the heterogeneous wetting regime was proposed by Cassie and Baxter [10]:

$$
\cos \theta_{C B}=f \cos \theta_{Y}+f-1
$$

If the roughness ratio, $r_{f}$, the ratio of the actual wetted area over the projected area is considered, equation (4) can be modified to the following form [11]:

$$
\cos \theta_{C B}=r_{f} f \cos \theta_{Y}+f-1
$$

where $f$ is the area fraction on the horizontal projected plane of the liquid-solid contact area over the total area of solid-liquid and liquid-gas contact. Equation (5) would become the same form with Wenzel's equation when $f=1$ and $r_{f}=r$. By equating equation (3) and equation (5), the critical contact angle theoretically used to separate the two wetting states can be calculated as [12]:

$$
\cos \theta_{C}=\frac{1-f}{r_{f} f-r}
$$

It should be noted that when $\theta_{C}>90^{\circ}$, both two wetting states exist. Then the homogeneous wetting state is preferable only if $\theta_{Y}<\theta_{C}$, otherwise the droplet stays at a heterogeneous wetting state, theoretically [13]. However, it has been observed that, even the Young's angle is smaller than the critical angle, the Cassie-Baxter wetting state can exist, which means that Wenzel and Cassie Baxter states may stay on the same specific surface at the same time [14$18]$.

Bormashenko E. reviewed the main experimental and theoretical approaches to wetting transitions in 2010 and 2015 respectively $[19,20]$. Experiments to study the wetting transitions were implemented by giving external factors such as pressure [21], initial velocity [22], evaporation of droplets [23], vibration [24], and electric field [25, 26]. And the role of gravity in wetting transitions was also discussed [11]. Neelesh A. Patankar [11] and Zu Y. et al [27] 
theoretically analyzed the wetting transition from Cassie-Baxter state to Wenzel state from the free energy point of view, and the energy barrier was discussed both in their work. Whyman G. et al. [28] theoretically investigated the interfacial free energy and discussed the irreversibility of Cassie-to-Wenzel transition. Ren W [29] computed the transition states, the energy barriers and the minimum energy paths for Cassie-to-Wenzel transition using the string method. G. Pashos et al. $[30,31]$ developed a numerical method to investigate the minimum energy paths and the free energy changes were presented in their works. S. Prakash et al. [32] studied the spontaneous recovery of superhydrophobicity on nanotextured surfaces using molecular simulations. Bico J. et al. [33] and Aurbach D. et al. [34] studied the Cassie impregnating state apart from the Cassie-Baxter state and Wenzel state, and Gibbs free energy curves of the three wetting states were presented. In their work the impregnating state was observed via vibration so that the liquid can impregnate the grooves outside of the droplet/solid interface. In this paper, we focus on the transition between the more regular Cassie-Baxter and Wenzel wetting states.

Wenzel's equation and Cassie and Baxter's equation can describe the stable wetting states on real rough surfaces to a great extent when the droplet size is much larger than the typical roughness scale. Nevertheless, there are still points of the theory of wetting states which are not fully understood. For instance, when a droplet stays in a stable wetting state, and how the transition between the two wetting states occurs [13]. It is crucial to understand the mechanism of wetting transition process for the design and manufacturing of devices with highly stable superhydrophobic surfaces. This paper focuses on the wetting transition process as well as the different wetting states on the simplest model, the square-post patterned surface from the free energy point of view.

\section{Theoretical analysis}

In the present study, the substrate patterned by square posts as the roughness surface is considered as shown in Figure 1, where a, b and $\mathrm{h}$ are the post width, post spacing, and post height respectively. It should be pointed out that the droplet size scale is much larger than the 
114 analysis can be conducted based on a single unit of patterned substrate with periodical pattern

115 and the Wenzel and Cassie-Baxter equations can be used for the calculation of the apparent 116 contact angles. In the presented pattern, $r_{f}$ equals to 1.

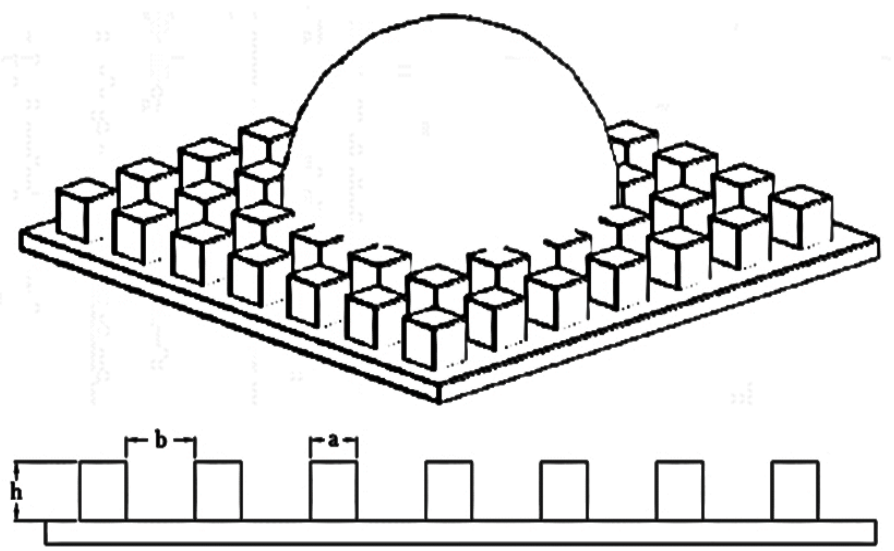

Figure 1 Structure of the micro roughness surface

\subsection{The model of net free energy}

All the parameters needed for the following theoretical analysis are presented in Figure 2 in three typical wetting state cases. Firstly, considering a droplet staying steady on a flat ideal surface as shown in Figure 2(a), the equilibrium free energy can be calculated as [13]:

$$
E_{Y}=S\left(\sigma_{S L}-\sigma_{S G}\right)+S^{\prime} \sigma_{L G}
$$

where $\mathrm{S}$ and $\mathrm{S}$ ' represent the solid/liquid interface area and the liquid/gas interface area respectively. Similarly, the equilibrium free energy equations for Cassie-Baxter and Wenzel states are:

$$
E_{C B}=S_{C B}\left(\sigma_{S L}^{\prime}-\sigma_{S G}\right)+S_{C B}^{\prime} \sigma_{L G}
$$

$$
E_{W}=S_{W}\left(\sigma_{S L}^{\prime}-\sigma_{S G}\right)+S_{W}^{\prime} \sigma_{L G}
$$

where $\sigma_{S L}^{\prime}$ is the equivalent free energy per unit area of the solid/liquid interfaces for both of the two states, while $S_{C B}$ and $S_{W}$ both represent the projected horizontal areas. Considering the equivalent surface tension, Young's equation can be applied into the heterogeneous and homogeneous wetting states: 
$136=\frac{\sigma_{S G}-\sigma_{S L}^{\prime}}{\sigma_{L G}}$

$138=\frac{\sigma_{S G}-\sigma_{S L}^{\prime}}{\sigma_{L G}}$

139 By combining the above equations, the free energy equations for Cassie-Baxter and Wenzel

140 states can be expressed as:

$$
\begin{aligned}
& E_{C B}=S_{C B}\left[f\left(\sigma_{S L}-\sigma_{S G}\right)+(1-f) \sigma_{L G}\right]+S_{C B}^{\prime} \sigma_{L G} \\
E_{W}= & S_{W} r\left(\sigma_{S L}-\sigma_{S G}\right)+S_{W}^{\prime} \sigma_{L G}
\end{aligned}
$$

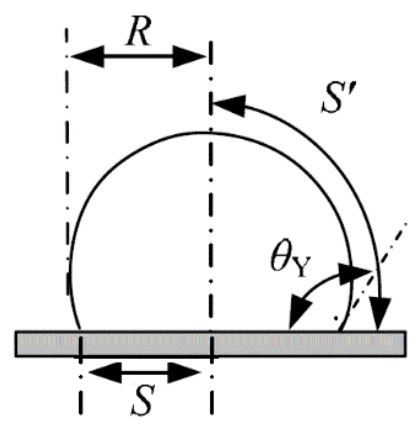

(a)

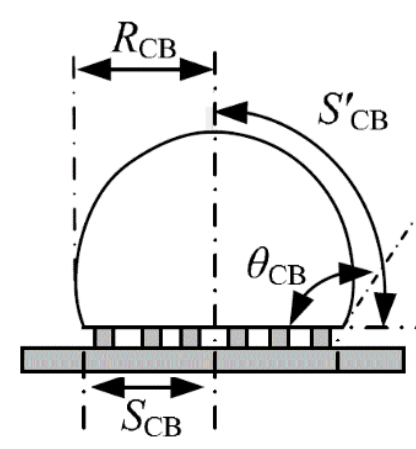

(b)

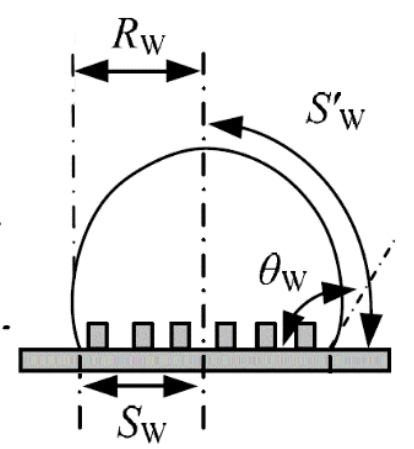

(c)

Figure 2 Parameters of the droplet in (a) flat surface (b) Cassie-Baxter state and (c) Wenzel state

\subsection{Cassie-to-Wenzel wetting transition}

\subsubsection{Without gravity effects}

152 Usually, the transition process from Cassie-Baxter state to Wenzel state can be easily observed,

153 however, the reverse process is hard to be achieved. Thus it is generally agreed that the wetting transition from Cassie-Baxter state to Wenzel state is irreversible [20]. Figure 3 shows the two main processes of wetting transition: (a) water starting to penetrate the posts intervals without touching the bottom surface; (b) water immersing the bottom surface. The position of the air pocket in Figure 3(b) can be neglected because the immersing-bottom process just lowers the 
158 free energy and does not hinder the transition regarding the following analysis. $\widetilde{E}_{C B}$ and $\tilde{E}_{W}$ 159 are used to represent the intermediate free energy of the droplet. According to equation (7), 160 there are:

161

$$
\begin{aligned}
& \tilde{E}_{C B}=\tilde{S}_{C B}\left\{\left[f+(r-1) \frac{h^{\prime}}{h}\right]\left(\sigma_{S L}-\sigma_{S G}\right)+(1-f) \sigma_{L G}\right\}+\tilde{S}_{C B}^{\prime} \sigma_{L G} \\
& \tilde{E}_{W}=\tilde{S}_{W}\left[r-(1-f) \frac{d^{\prime}}{d}\right]\left(\sigma_{S L}-\sigma_{S G}\right)+\left[\tilde{S}_{W}^{\prime}+\tilde{S}_{W}(1-f) \frac{d^{\prime}}{d}\right] \sigma_{L G}
\end{aligned}
$$

163 When $h^{\prime}$ and $d^{\prime}$ are on their extreme values $h$ and $d$, the critical free energy states can be 164 achieved:

$$
\begin{gathered}
\hat{E}_{C B}=\hat{S}_{C B}\left\{[f+(r-1)]\left(\sigma_{S L}-\sigma_{S G}\right)+(1-f) \sigma_{L G}\right\}+\hat{S}_{C B}^{\prime} \sigma_{L G} \\
\hat{E}_{W}=\hat{S}_{W}[r-(1-f)]\left(\sigma_{S L}-\sigma_{S G}\right)+\left[\hat{S}_{W}^{\prime}+\hat{S}_{W}(1-f)\right] \sigma_{L G}
\end{gathered}
$$

167

168

169

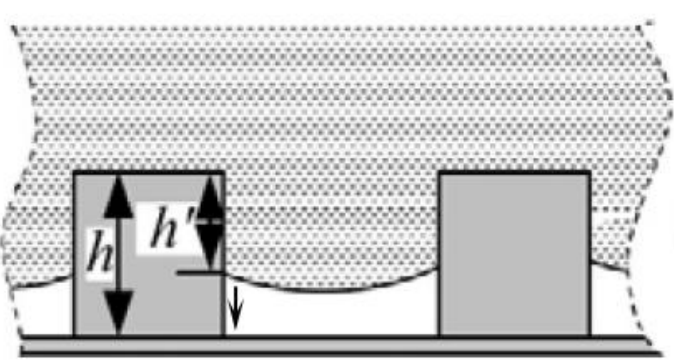

(a)

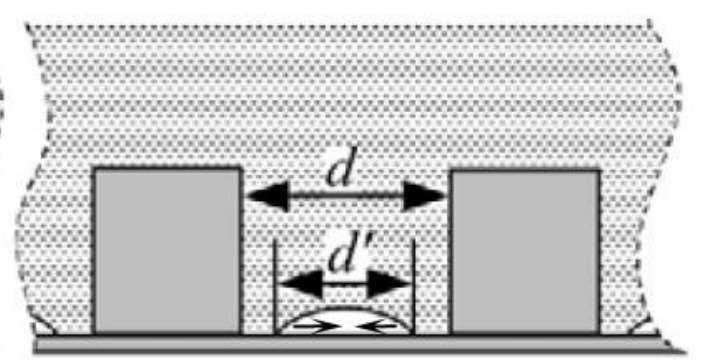

(b)

Figure 3 Intermediate states for transition (a) water starting to penetrate the posts intervals without touching the bottom surface (b) water immersing the bottom surface

It has been proved that the differences of the liquid/gas area and the droplet bottom projected area when transition happens are negligible owing to the much larger size scale compared to that of the surface roughness, which means $\hat{S}_{C B} \approx S_{C B}, \hat{S}_{W} \approx S_{W}, \hat{S}_{C B}^{\prime} \approx S_{C B}^{\prime}$ and $\hat{S}_{W}^{\prime} \approx S_{W}^{\prime}$ $[11,35]$. Hence there is $\widehat{E}_{C B}=\widehat{E}_{W}=E_{C r}$ for the same droplet in different states. And the energy barriers for the two transitions process can be calculated as:

$$
\begin{aligned}
E_{\text {bar }}^{C B-C r} & =\widehat{E}_{C B}-E_{C B}=S_{C B}(r-1)\left(\sigma_{S L}-\sigma_{S G}\right) \\
E_{\text {bar }}^{\mathrm{Cr}-W} & =E_{W}-\hat{E}_{W}=S_{W}(f-1)\left(\sigma_{L G}-\sigma_{S L}+\sigma_{S G}\right)
\end{aligned}
$$

For hydrophobic surfaces, i.e. $\theta_{Y}>90^{\circ}$, according to the Young's equilibrium equation, there are $\sigma_{S L}-\sigma_{S G}>0$ and $\sigma_{L G}-\sigma_{S L}+\sigma_{S G}>0$, therefore 
187 Correspondingly, for hydrophilic water, i.e. $\theta_{Y}<90^{\circ}$, there are $\sigma_{S L}-\sigma_{S A}<0$ and $\sigma_{L A}-$ $\sigma_{S L}+\sigma_{S A}>0$, therefore

193 Whether the transition can occur depends on the sign of the differential of free energy at the

194 beginning of the process. For transitions from Cassie-Baxter state to Wenzel state and the reverse, the free energy differentials can be given as:

Consequently, without considering the gravity effect or other external forces, the free energy curves can be drawn in Figure 4. Figure 4(a) and Figure 4(b) show the case of $\theta_{Y}>90^{\circ}$, when two main roughness surface features exist: (a) $90^{\circ}<\theta_{Y}<\theta_{C}, E_{C B}>E_{W}$, according to the assumption that the equilibrium state occurs when the free energy is minimized [11], both of the two states exist, however, the Wenzel state is stable while Cassie-Baxter state is not; (b) $\theta_{Y}>\theta_{C}, E_{W}>E_{C B}$, the droplet would stay in the Cassie-Baxter state, but may not in the Wenzel state and the analysis relating to this is in the next section. This means the energy barrier

205 always exists for the Cassie-to Wenzel transition for $\theta_{Y}>90^{\circ}$. In addition, $\left.\frac{\delta E^{C B-W}}{\delta h^{\prime}}\right|_{h^{\prime}=0}>0$

206 denotes that the transition processes cannot happen spontaneously without any external stimuli

207 triggering event. Figure 4(c) indicates that Cassie-Baxter state cannot be achieved if $\theta_{Y}<90^{\circ}$,

208 when $E_{C B}>E_{W}$ and $\left.\frac{\delta E^{C B-W}}{\delta h^{\prime}}\right|_{h^{\prime}=0}<0$ thus the droplet can only stays at the Wenzel state. It 
should be noted that all the free energy curves presented in this paper are qualitatively constructed because there exist uncertainties for the wetting transitions, for example, the bottom

211 droplet surface moving down along the posts is not definitely horizontal and when and which part of the droplet touches the bottom solid surface first is indeterminate.

213

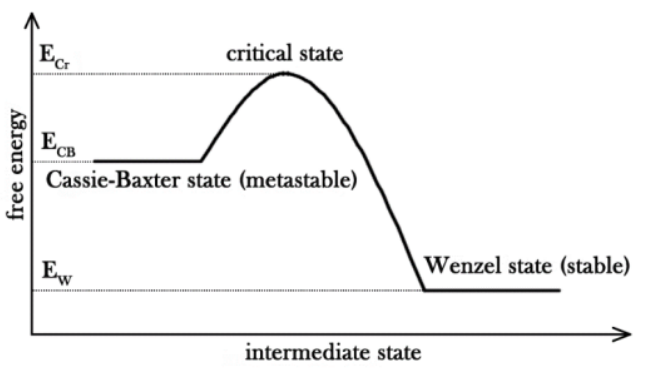

(a)

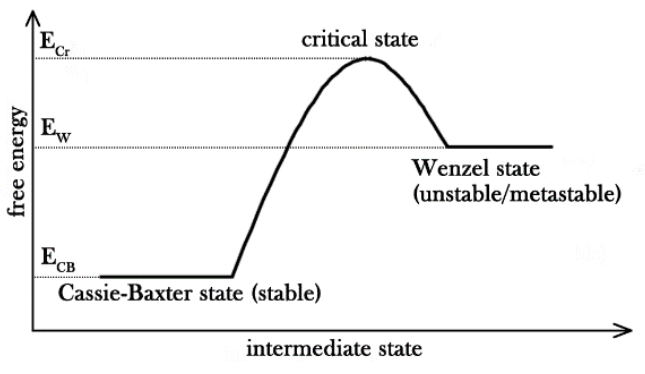

(b)

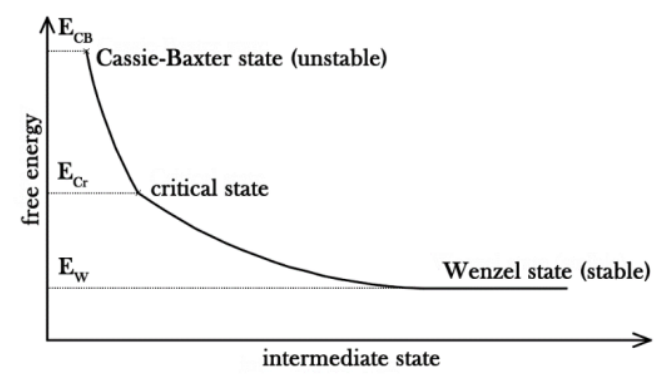

(c)

Figure 4 Free energy curves without gravity effect for (a) $90^{\circ}<\theta_{Y}<\theta_{C}$ (b) $\theta_{Y}>\theta_{C}$ and (c) $\theta_{Y}<90^{\circ}$

\subsubsection{With gravity effects}

The gravity does not affect the shape and the wetting state of a droplet significantly when the drop radius is much smaller than $\left(\sigma_{L A} / \rho g\right)^{1 / 2}$. However, its influence on transition may be nonnegligible [11]. When a droplet transits from the Cassie-Baxter state to the Wenzel state, the potential energy of gravity $E_{G}$ declines as well. Since the potential energy change occurs along with the transition process between Cassie-Baxter state and the critical state when the droplet is about to immerse the air pockets completely but have not yet reached the bottom 
surface, the energy curves can be modified by adding the potential energy change of which the

230 sign is negative. When $\theta_{Y}<90^{\circ}$, the energy curve is similar to Figure 4(c), where the energy 231 change is monotonous. However, for $\theta_{Y}>90^{\circ}$, one more case appears as shown in figure 5:

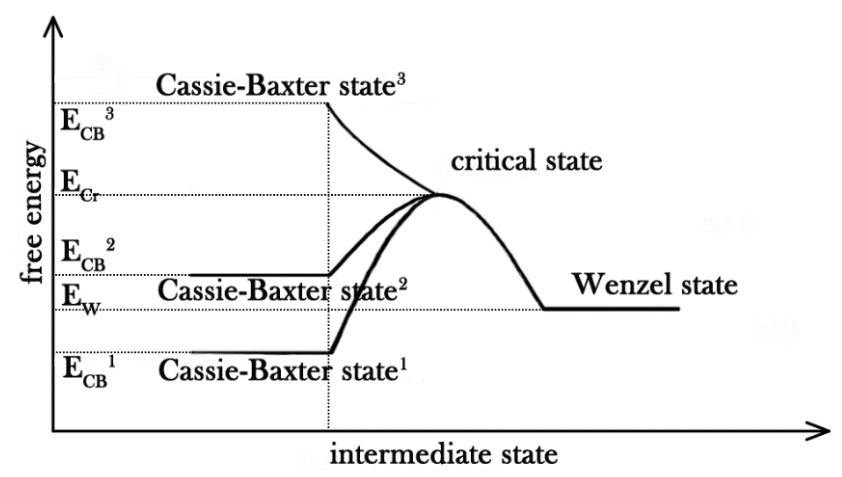

Figure 5 Energy curves with gravity effect for $\theta_{Y}>90^{\circ}$

Figure 5 shows the extra curve of case 3 when considering the gravity effect with a monotonous energy change, which denotes that the transition can occur spontaneously. In this case, the source of potential energy change $\Delta E_{G}$ can overcome the energy barrier $E_{b a r}^{C B-C r}$, and the conclusion is the same with that from Patankar, N. A. [11] which is achieved via comparing the theoretical analysis with experimental data from Yoshimitsu et al [26].

\subsection{Discussion about the irreversibility of wetting transition}

As mentioned above, it is generally thought that the transition from Cassie-Baxter state to Wenzel state is irreversible. From figure 5 it can be seen that the gravity potential energy can decrease the energy barrier $E_{\text {bar }}^{C B-W}$ or even overcome it. Besides, other external stimuli such as initial velocity, pressure and vibration can also be also used to overcome the energy barrier. Therefore, in most cases the Cassie-to-Wenzel transition is easier to be achieved, and more attention is paid on this transition due to its importance to superhydrophobic surfaces development.

Without considering the gravity effect, the reverse Wenzel-to-Cassie transition would take a different route. It is reasonable to assume the transition happens on the bottom from the vicinity 
of the gas-liquid-solid triple line, since air cannot be generated from the void, as shown in

253 Figure 6. Therefore, the triple lines may move simultaneously in the horizontal and vertical 254 directions. The energy decreased as solid-liquid contact area decreases may overcome the energy increased as liquid-gas contact area and solid-gas contact area increase, and if not, the reversible transition cannot occur spontaneously. Figure 6 presents the different routes of wetting transitions. It should be noticed that the reverse energy change may not be monotonous in Figure 7(a) in the case that the droplet is separated from the bottom but the vertical process has not finished yet, and in Figure 7(b) the critical Young's angle for the Wenzel-to-Cassie transition may not be the same with the critical angle in Equation (6).

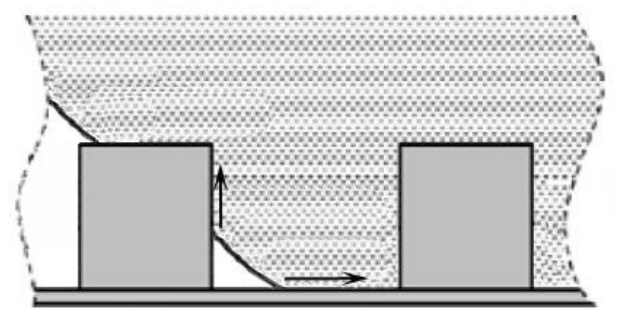

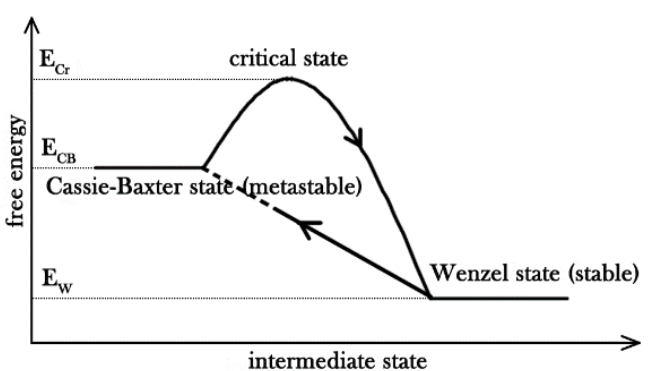

(a)

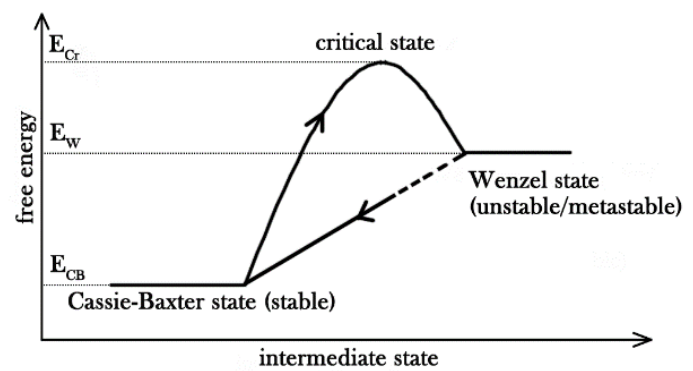

(b)

Figure 7 Free energy curves without gravity effect for (a) $90^{\circ}<\theta_{Y}<\theta_{C}$

(b) $\theta_{Y}>\theta_{C}$

Gravity potential can be considered as a part of the energy barrier needed to overcome.

268 It is much more difficult to trigger the reverse transition than the Cassie-to-Wenzel transition due to the different transition routes, which can explain the irreversibility of wetting transition. Experiments to achieve the reverse transition were carried out by heating the substrate [36] or transmitting a short pulse of electrical current [26], and 
both of the two experiments appeared to be conducted by the evaporation of the droplet in the vicinity of their gas-liquid-solid triple line, changing liquid phase to vapor phase to break the reverse energy barrier and complete the reverse transition. Thus a metastable Cassie-Baxter wetting state can be achieved as shown in Figure 7(a).

The energy curves shown in Figure 7 can be very helpful to understand the wetting transition mechanism and develop superhydrophobic surfaces. Some surfaces with topographic features involving specialized geometries such as inverse trapezoidal [37], T-shape [38] and serif-T [39] are the typical examples to impede Cassie-to-Wenzel wetting transition by increasing the energy barrier during Cassie-to-critical process, namely raising the critical state energy in Figure 7. However, few papers were found to focus on the critical-to-Wenzel process, which could also be a crucial factor to affect wetting transition because no matter how high the critical state energy is the Cassie-toWenzel transition can be finished when the energy barrier is overcame by external forces. Such work relating to the bottom surface as well as the critical-to-Wenzel process will be investigated in the future.

\section{Numerical simulation}

In this section, the simulation with a phase field lattice Boltzmann method with large density ratio developed by Y. Q. Zu [40] is implemented to study the wetting states. As shown in Figure 8 , a spherical water droplet with the initial radius $30 \mu \mathrm{m}$ is placed on the patterned surfaces in different wetting states and different Young's contact angles. In the simulation, $a=d=h=$ $5 \mu m$, and the critical Young's angle $\theta_{C}=115.4^{\circ}$ calculated via equation (6). Young's angles $\theta_{Y}$ of $105^{\circ}$ and $130^{\circ}$ are set for a Wenzel preferable and a Cassie-Baxter preferable states. The water/gas properties are set naturally as: $\rho_{L}=1000 \mathrm{~kg} / \mathrm{m}^{3}, \rho_{G}=1.204 \mathrm{~kg} / \mathrm{m}^{3}, \mu_{L}=$ $1 \times 10^{-3} \mathrm{~kg} /(\mathrm{m} \cdot \mathrm{s}), \mu_{G}=1.9 \times 10^{-5} \mathrm{~kg} /(\mathrm{m} \cdot \mathrm{s})$ for the density and dynamic viscosity, respectively. Gravity is not considered in the simulation because it can be seen as an external force to trigger the transition. 
301 After evolving for $2,000,000 \delta_{t}(20 \mathrm{~ms})$, where $\delta_{t}$ is the time step of LBM, all the droplets 302 go stable and the shapes of the droplets do not change anymore. Eventually, for $\theta_{Y}=105^{\circ}$, 303 both Wenzel state and Cassie-Baxter state can be achieved with the apparent contact angles $304119^{\circ}$ and $137^{\circ}$, while for $\theta_{Y}=130^{\circ}$, there is just the Cassie-Baxter state left, with $\theta_{C B}=$ $305158^{\circ}$. The apparent contact angles calculated by equation (3) and equation (5) are $121.2^{\circ}$, $306144.6^{\circ}$ and $155.6^{\circ}$ respectively, which are close to the present simulation data.

307

308

309

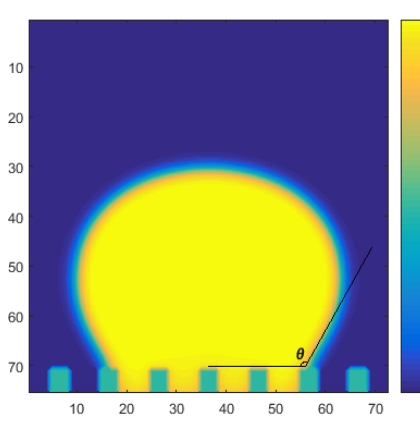

(a)

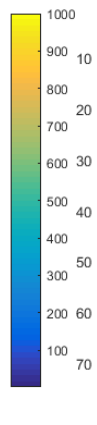

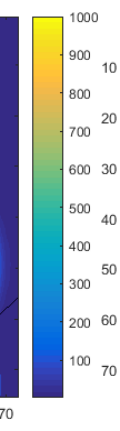

(b)

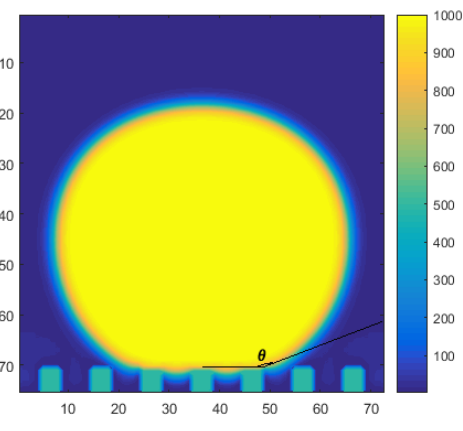

(c)

Figure 8 Wetting states for different Young's angles, $\theta_{C}=115.4^{\circ}, t=2,000,000 \delta_{t}$ (a) $\theta_{Y}=105^{\circ}$, Wenzel state (b) $\theta_{Y}=105^{\circ}$, Cassie-Baxter state (c) $\theta_{Y}=130^{\circ}$, Cassie-Baxter state

The dynamic wetting transition on an intrinsically hydrophilic surface with $\theta_{Y}=75^{\circ}$ is simulated, as shown in Figure 9. The stable Cassie-Baxter state cannot be observed as the transition occurs spontaneously without any external forces.

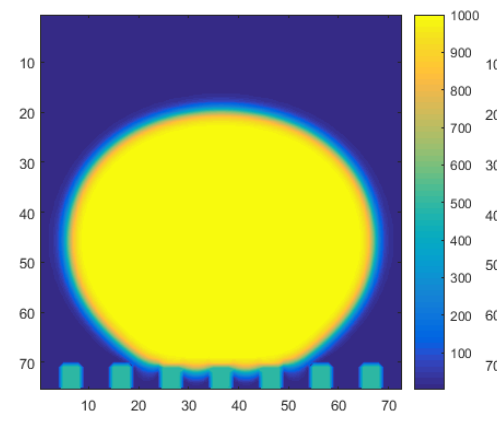

(a)

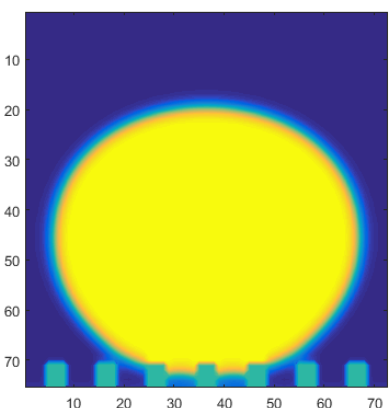

(b)

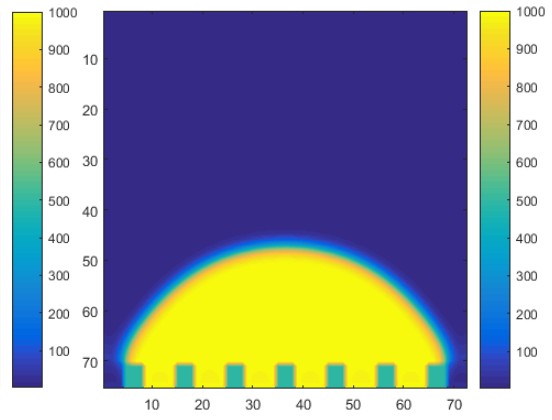

(c)

Figure 9 Water spreading on the patterned surface for $\theta_{Y}=75^{\circ}$ (a) $t=0 \delta_{t}$ (b) $t=$

$$
40,000 \delta_{t} \text { (c) } t=700,000 \delta_{t}
$$

Figure 10 shows the Wenzel-Cassie transition process of the droplet with Young's contact angle 

that the transition occurs on the patterned surface from the vicinity of the three phase line.

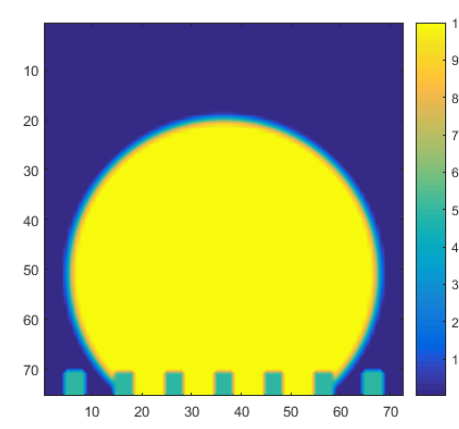

(a)

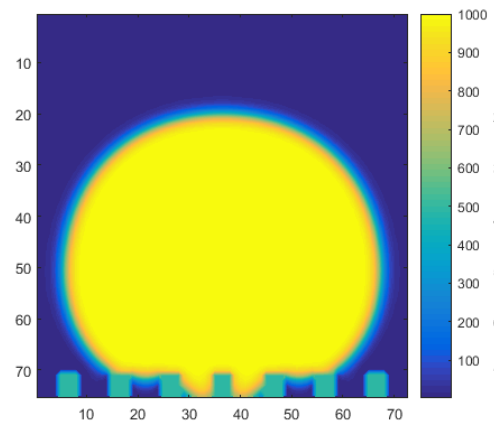

(d)

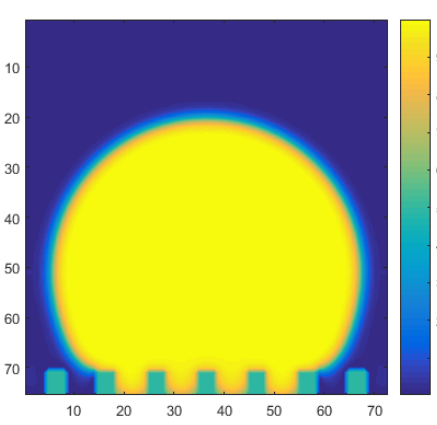

(b)

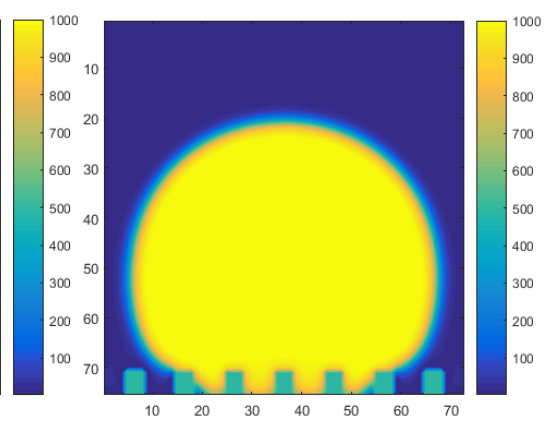

(c)

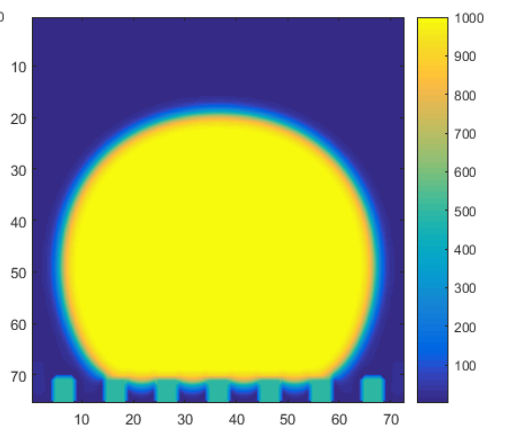

(e)

Figure 10 The Wenzel-Cassie transition for $\theta_{Y}=130^{\circ}$ (a) $t=0 \delta_{t}$ (b) $t=10,000 \delta_{t}$

(c) $t=60,000 \delta_{t}$

(d) $t=130,000 \delta_{t}$

(e) $t=170,000 \delta_{t}$

The simulations are in good agreement with the proposed energy curves. The droplet in CassieBaxter state in Figure 8(b) has a higher free energy compared to the droplet in Wenzel state, but it can keep steady, which means there is an energy barrier existing between the two wetting states. In addition, the simulation excludes the influence from the roughness of much smaller order of sizes which might be a factor to determine the two wetting state in experimental studies. When the intrinsic contact angle is $75^{\circ}$, smaller than $90^{\circ}$, there is no stable Cassie-Baxter state observed. The transition occurs spontaneously without any external forces, which means there is no energy barrier between the two wetting states. For a higher inherent contact angle $\theta_{Y}=$ $130^{\circ}$ the Wenzel state in the simulation is unstable and the Wenzel-to-Cassie occurs spontaneously, confirming the reverse transition route in Figure 7(b). However, when testing some cases in which the Young's angles are between $130^{\circ}$ and the critical angle, the reverse transition cannot be observed. This means the critical contact angle for Wenzel-to-Cassie 
transition is not the same as the one determining the same energies of Cassie-Baxter state and

341 Wenzel state.

\section{$344 \quad 4$ Conclusions}

345 In this paper, the wetting transitions for a droplet on a square-post patterned surface are

346 theoretically analyzed. Numerical simulations with a phase field lattice Boltzmann method

347 were carried out, and the results show good agreement with the theoretical analysis. The main

348 finding of this work is that the energy curves during wetting transitions are proposed for Cassie-

349 to-Wenzel transition together with the reverse transition via the theoretical analysis of the free

350 energy changes during the transitions processes. The energy curves give a clear description of

351 the conditions in which the transitions occur and the energy barriers exist for both transition

352 processes. Gravity effect for wetting transition is considered, and the energy curves illustrate

353 that the gravity can be a driving force to trigger the transition. The irreversibility is discussed

354 based on the energy curves presented. The Wenzel-to-Cassie transition can occur spontaneously

355 only if the inherent contact angle is large enough. It can also be concluded from the curves that

356 different routes of the Cassie-to-Wenzel transition and the reverse transition are the main reason

357 for the irreversibility of wetting transitions. The work is based on the regular square-post

358 patterned surface, which is also the basis of most complicated rough surfaces. Therefore the

359 presented energy curves can be very helpful to understand the mechanism of complex wetting

360 phenomena.

\section{Acknowledgement}

364 The authors would like to acknowledge the financial support of this work by the doctoral degree

365 scholarship of China Scholarship Council and the University of Nottingham, UK. 
1. Li L, Liu X, Dai X J, et al. (2013) Surface wetting processing on BNNT films by selective plasma modes. Chin Sci Bull 58:3403-3408

2. Yao J, Wang J, Yu Y, et al. (2012) Biomimetic fabrication and characterization of an artificial rice leaf surface with anisotropic wetting. Chin Sci Bull 57:2631-2634

3. Yao Z, Hao P, Zhang X, et al. (2012) Static and dynamic characterization of droplets on hydrophobic surfaces. Chin Sci Bull 57:1095-1101

4. Yan Y (2009) Physical and numerical modelling of biomimetic approaches of natural hydrophobic surfaces. Chin Sci Bull 54:541-548

5. Gao N, Yan Y, Chen X, et al. (2012) Nanoparticle-induced morphology and hydrophilicity of structured surfaces. Langmuir 28:12256-65

6. Huang $\mathrm{Y}, \mathrm{Hu} \mathrm{Y}$, Zhu C, et al. (2016) Long-Lived Multifunctional Superhydrophobic Heterostructure Via Molecular Self-Supply. Adv Mater Interfaces 3:1500727

7. Koch K, Bhushan B, and Barthlott W (2009) Multifunctional surface structures of plants: an inspiration for biomimetics. Prog Mater Sci 54:137-178

8. Young $T$ (1805) An essay on the cohesion of fluids. Philos Trans R Soc London 95:65-87

9. Wenzel R N (1949) Surface Roughness and Contact Angle. J Phys Chem 53:1466-1467

10. Cassie A and Baxter S (1944) Wettability of porous surfaces. Trans Faraday Society 40:546-551

11. Patankar N A (2004) Transition between superhydrophobic states on rough surfaces. Langmuir 20:7097-7102

12. Gao N and Yan Y (2009) Modeling Superhydrophobic Contact Angles and Wetting Transition. J

13. Yan $Y$, Gao N, and Barthlott W (2011) Mimicking natural superhydrophobic surfaces and grasping the wetting process: A review on recent progress in preparing superhydrophobic surfaces. Adv Colloid Interface Sci 169:80-105

14. Marmur A and Bittoun E (2009) When Wenzel and Cassie are right: reconciling local and global considerations. Langmuir 25:1277-1281

15. Marmur A (2008) From hygrophilic to superhygrophobic: theoretical conditions for making high-contact-angle surfaces from low-contact-angle materials. Langmuir 24:7573-7579

16. Ran C, Ding G, Liu W, et al. (2008) Wetting on nanoporous alumina surface: transition between Wenzel and Cassie states controlled by surface structure. Langmuir 24:9952-9955

17. Jung Y C and Bhushan B (2007) Wetting transition of water droplets on superhydrophobic patterned surfaces. Scripta Mater 57:1057-1060

18. Koishi T, Yasuoka K, Fujikawa S, et al. (2009) Coexistence and transition between Cassie and Wenzel state on pillared hydrophobic surface. Proc Natl Acad Sci U S A 106:8435-40

19. Bormashenko E (2010) Wetting transitions on biomimetic surfaces. Philos Trans A Math Phys Eng Sci 368:4695-711

20. Bormashenko E (2015) Progress in understanding wetting transitions on rough surfaces. Adv Colloid Interface Sci 222:92-103

$40922 . \quad J u n g ~ Y C$ and Bhushan B (2009) Dynamic effects induced transition of droplets on biomimetic 
superhydrophobic surfaces. Langmuir 25:9208-9218

23. McHale G, Aqil S, Shirtcliffe N, et al. (2005) Analysis of droplet evaporation on a superhydrophobic surface. Langmuir 21:11053-11060

24. Bormashenko E, Pogreb R, Whyman G, et al. (2007) Resonance Cassie-Wenzel wetting transition for horizontally vibrated drops deposited on a rough surface. Langmuir 23:1221712221

25. Bahadur V and Garimella S V (2008) Electrowetting-based control of droplet transition and morphology on artificially microstructured surfaces. Langmuir 24:8338-8345

26. Krupenkin T N, Taylor J A, Wang E N, et al. (2007) Reversible wetting-dewetting transitions on electrically tunable superhydrophobic nanostructured surfaces. Langmuir 23:9128-9133

27. Zu Y and Yan Y (2016) Single Droplet on Micro Square-Post Patterned Surfaces-Theoretical Model and Numerical Simulation. Sci Rep 6:

28. Whyman G and Bormashenko E (2012) Wetting transitions on rough substrates: General considerations. J Adhes Sci Technol 26:207-220

29. Ren W (2014) Wetting transition on patterned surfaces: transition states and energy barriers. Langmuir 30:2879-85

30. Pashos G, Kokkoris G, and Boudouvis A G (2015) Minimum energy paths of wetting transitions on grooved surfaces. Langmuir 31:3059-68

31. Pashos G, Kokkoris G, Papathanasiou A G, et al. (2016) Wetting transitions on patterned surfaces with diffuse interaction potentials embedded in a Young-Laplace formulation. J Chem Phys 144:034105

32. Prakash S, Xi E, and Patel A J (2016) Spontaneous recovery of superhydrophobicity on nanotextured surfaces. Proc Natl Acad Sci 201521753

33. Bico J, Thiele U, and Quéré D (2002) Wetting of textured surfaces. Colloids Surf Physicochem Eng Aspects 206:41-46

34. Bormashenko E, Pogreb R, Stein T, et al. (2008) Characterization of rough surfaces with vibrated drops. Phys Chem Chem Phys 10:4056-4061

35. Zu Y, Yan Y, Li J, et al. (2010) Wetting behaviours of a single droplet on biomimetic micro structured surfaces. J Bionic Eng 7:191-198

36. Liu G, Fu L, Rode A V, et al. (2011) Water droplet motion control on superhydrophobic surfaces: exploiting the Wenzel-to-Cassie transition. Langmuir 27:2595-2600

37. Im M, Im H, Lee J-H, et al. (2010) A robust superhydrophobic and superoleophobic surface with inverse-trapezoidal microstructures on a large transparent flexible substrate. Soft Matter 6:1401-1404

38. Cai T-m, Jia Z-h, Yang H-n, et al. (2016) Investigation of Cassie-Wenzel Wetting transitions on microstructured surfaces. Colloid Polym Sci 294:833-840

39. Liu T L and Kim C J (2014) Repellent surfaces. Turning a surface superrepellent even to completely wetting liquids. Science 346:1096-100

40. Zu Y Q and Yan Y Y (2011) Lattice Boltzmann method for modelling droplets on chemically heterogeneous and microstructured surfaces with large liquid-gas density ratio. IMA J Appl Math 76:743-760 